\title{
Microvascular flow, clinical illness severity and cardiovascular function in the preterm infant
}

\author{
M J Stark, V L Clifton and I M R Wright
}

Arch Dis Child Fetal Neonatal Ed 2008 93: F271-F274 originally published online February 19, 2008

doi: 10.1136/adc.2007.123539

Updated information and services can be found at:

http://fn.bmj.com/content/93/4/F271.full.html

Topic collections Articles on similar topics can be found in the following collections
Epidemiologic studies (23563 articles)
Echocardiography (1617 articles)
Hypertension (11754 articles)
Child health (26025 articles)
Infant health (4399 articles)
Neonatal health (1873 articles)
Radiology (14736 articles)
Clinical diagnostic tests (18613 articles)
Radiology (diagnostics) (9749 articles)

Notes

To order reprints of this article go to:

http://fn.bmj.com/cgi/reprintform

To subscribe to Archives of Disease in Childhood - Fetal and Neonatal Edition go to: http://fn.bmj.com/subscriptions 


\title{
Microvascular flow, clinical illness severity and cardiovascular function in the preterm infant
}

\author{
M J Stark, ${ }^{1}$ V L Clifton, ${ }^{1}$ I M R Wright ${ }^{1,2}$
}

\begin{abstract}
${ }^{1}$ Mother and Babies Research Centre, Hunter Medical Research Institute, University of Newcastle, Newcastle, NSW, Australia; ${ }^{2}$ Neonatal Intensive Care Unit, The John Hunter Children's Hospital, Newcastle NSW, Australia

Correspondence to: Dr I Wright, Kaleidoscope Neonatal Intensive Care Unit, John Hunter Children's Hospital, Newcastle, NSW 2310, Australia; Ian.Wright@ hnehealth.nsw.gov.au
\end{abstract}

Accepted 28 January 2008 Published Online First

19 February 2008

\section{ABSTRACT}

Objectives: To characterise the relationships between peripheral microvascular blood flow and measures of physiological and cardiovascular function in preterm infants in the immediate newborn period.

Design: Prospective observational cohort study.

Setting: Tertiary neonatal intensive care unit, New South Wales, Australia.

Patients: Ninety-six preterm neonates (24-36 weeks' gestation) admitted to the neonatal intensive care unit. Main outcome measure: Relationship between laser Doppler-derived basal microvascular blood flow, functional echocardiographic measurements of cardiovascular status, mean arterial blood pressure and clinical illness severity at 24,72 and $120 \mathrm{~h}$ of age.

Results: At 24 h of age, multiple linear regression revealed a significant positive relationship, independent of gestational age, between baseline microvascular blood flow and clinical risk index for babies (CRIB II) score $\left(r^{2}=0.442\right)$. Microvascular blood flow was inversely related to mean arterial blood pressure $\left(r^{2}=-0.563\right)$, and correlated positively with left ventricular output $\left(r^{2}=0.435\right)$. Microvascular blood flow continued to exhibit a significant inverse relationship with mean arterial blood pressure $\left(r^{2}=-0.4\right)$ at $72 \mathrm{~h}$ of age, but by $120 \mathrm{~h}$ no significant relationships were evident.

Conclusions: This is the first study to show that baseline microvascular blood flow in premature infants exhibits significant relationships with clinical illness severity and cardiovascular function in the immediate postnatal period. The effects of temporal and functional changes in the microvasculature on cardiovascular adaptation warrant further detailed study.

Peripheral microvascular blood flow in newborn infants has been studied using a range of experimental tools. ${ }^{1-3}$ Most recently, laser Doppler flowmetry has been shown to be particularly useful in studying rapid changes in the skin microcirculation of the newborn infant. ${ }^{4}$ Studies of neonatal vasoregulation are of clinical relevance. Peripheral microvascular blood flow is subjected to considerable change in the first days of extrauterine life, a period of marked circulatory vulnerability in preterm infants. Myogenic and neural control of skin blood flow must be rapidly established after birth to allow appropriate thermoregulation in the newborn..$^{5}$ In addition, alterations in microvascular function play an important role in septicaemia, ${ }^{6}$ neonatal polycythaemia, ${ }^{7}$ and cardiovascular adaptation after delivery. ${ }^{8}$

It has long been established, using the technique of laser Doppler flowmetry and others, that the ability to control skin blood flow is well developed in neonates, including preterm infants. ${ }^{39}$ However, abnormal regulation of peripheral vascular resistance, producing inappropriate microvascular vasodilatation, could contribute to the development of circulatory compromise after preterm birth. Despite evidence from the animal literature ${ }^{10}$ and observational data in human neonates, ${ }^{11}$ the relationships between peripheral microvascular blood flow and measures of neonatal physiological and cardiovascular stability in the immediate newborn period have not been characterised. This study was undertaken to characterise the relationship between laser Doppler-derived basal peripheral microvascular blood flow, clinical illness severity, and measures of cardiovascular function.

\section{MATERIALS AND METHODS Subjects}

Ninety-six preterm infants (24-36 weeks' gestation) were studied during the first 5 days of life. Hypoxic ischaemic encephalopathy, congenital malformations, chromosomal disorders or known congenital infection excluded admission to this study. Recruitment was stratified to infants aged 28 weeks or less and preterm infants aged 29 36 weeks, in order to avoid severity effects being lost in the potentially larger numbers of more mature infants.

\section{Microvascular studies}

Microvascular laser Doppler assesses the motion in blood vessels of the peripheral microvasculature and skin tissues. ${ }^{12}$ Low-intensity laser light is reflected from moving blood cells in the skin circulation to allow measurement of blood flow. We used the Periflux 5001 Laser Doppler (Perimed $\mathrm{AB}$, Järfälla, Sweden) with a single temperatureregulated probe sited on the lateral aspect of the lower limb. All laser Doppler studies were performed by a single investigator (MS). The intrastudy coefficient of variation $((\mathrm{SD} /$ mean $) \times 100 \%)$ for baseline blood flow measurement over 10 individual subjects had a mean of $14.8 \%$ (range $6.2-22.8 \%)$.

\section{Experimental protocol}

Investigations were performed with a temperaturecontrolled laser Doppler probe set at $34^{\circ} \mathrm{C}$. Studies were not performed within $1 \mathrm{~h}$ of an enteral feed. Microcirculatory recordings were performed at times when the infant was lying supine quietly. Laser Doppler studies were performed at 24, 72 and $120 \mathrm{~h}$ of age.

Peripheral microvascular blood flow was recorded for $5 \mathrm{~min}$ before a standard provocation to allow comparison between different studies and subjects, as previously described. ${ }^{13}$ After estimation 
Table 1 Clinical characteristics of 96 preterm neonates

\begin{tabular}{ll}
\hline Gestational age (weeks) & $29(27-32)$ \\
Pregnancy-induced hypertension & $14(14.5)$ \\
Gestational diabetes & $7(7.3)$ \\
Vaginal delivery & $48(50)$ \\
Birth weight (g) & $1239(872-1803)$ \\
Small for gestational age & $26(27)$ \\
Maternal smoking & $18(19)$ \\
5 min Apgar score & $9(7-9)$ \\
CRIB II score & $9(4-12)$ \\
Total days oxygen & $2(0-11)$ \\
Total days IMV & $0(0-25.5)$ \\
Total days CPAP & $103(15-343)$ \\
Grade 3/4 IVH & $5(5.2)$ \\
Died & $8(8.3)$
\end{tabular}

Values are either number (\%) or median (interquartile range). Deaths are those occurring in first 2 weeks of life.

CRIB II, clinical risk index for babies; CPAP, continuous positive airway pressure; IMV, intermittent mandatory ventilation; IVH, intraventricular haemorrhage.

of basal blood flow, lower limb blood flow was occluded using a sphygmomanometer to produce a short period of absent flow. This allowed biological zero to be obtained in each experiment. This zero was subtracted from the blood flow in each experiment. ${ }^{13}$ Peripheral microvascular blood flow recordings were analysed off line using custom software (Perisoft 2.1; Perimed AB). Only recording sequences free from movement artefacts were analysed.

\section{Cardiovascular studies}

Mean arterial blood pressure, measured by in-dwelling arterial catheter where possible, was determined from averaged serial blood pressure readings over a $30 \mathrm{~min}$ period immediately before the laser Doppler recording.

Functional echocardiographic studies were performed by a sole investigator (MS) immediately after laser Doppler recording. An Acuson Aspen ultrasound machine (Acuson Corp, Mountain View, California, USA) incorporating colour flow, pulsed-wave and continuous-wave Doppler was used. Structural normality of the heart was established during the course of the scan. All studies were recorded to optical disc for later review and performance of measurements. Left and right ventricular output, ductal patency, superior vena caval (SVC) blood flow, and systemic vascular resistance were calculated as previously described. ${ }^{14}$

\section{Clinical illness severity}

Clinical illness severity was quantified using the clinical risk index for babies (CRIB II). ${ }^{15}$

\section{Statistical methods}

The data are described as median (interquartile range). Differences between groups were analysed by Mann-Whitney $U$ test. The data were not normally distributed and were therefore log-transformed for further analysis. Pearson $r$ was used for correlations between measures of peripheral microvascular blood flow, cardiovascular function, gestation and clinical illness severity. As gestation was found to correlate with multiple measures of cardiovascular function and illness severity, multiple linear regression was used to assess the contribution of peripheral blood flow independent of gestation. $p \leqslant 0.05$ was considered significant.
Informed consent was obtained from the mothers of the infants before the investigation, and the study protocol was approved by the human ethics committees at John Hunter Hospital and the University of Newcastle.

\section{RESULTS}

Ninety-six preterm neonates were studied and their clinical characteristics are shown in table 1. The median (range) gestational age was 29 (24-36) weeks and birth weight was 1239 (430-3035) g. Infants of $\leqslant 32$ weeks' gestation had a median CRIB II score of 9 (2-16).

\section{Peripheral microvascular blood flow, clinical illness severity and cardiovascular function}

At $24 \mathrm{~h}$ of age, a significant positive relationship, independent of gestational age, was revealed between baseline microvascular blood flow and CRIB II score $\left(r^{2}=0.442, p<0.001\right.$; fig 1$)$. In addition, in infants of $\leqslant 28$ weeks' gestation, at highest risk of death after preterm delivery, baseline microvascular blood flow was significantly higher in those infants who died within the first 72 h of life (median (IOR): 224.7 (95.6-302.2) vs 34.1 (17.966.7) perfusion units (PU), $p=0.001$; fig 2) compared with those who survived. There was no difference in incidence of early culture positive sepsis between these infants.

Baseline peripheral microvascular blood flow exhibited significant relationships with measures of cardiovascular function at $24 \mathrm{~h}$. Laser Doppler microvascular flow was inversely related to mean arterial blood pressure $\left(r^{2}=-0.563, p<0.001\right.$; fig 3), calculated systemic vascular resistance (left ventricular output/mean arterial blood pressure) $\left(r^{2}=-0.479, p<0.001\right)$, and positively related to left ventricular output $\left(r^{2}=0.435\right.$, $\mathrm{p}<0.001$ ), independent of gestational age. No relationship was evident between baseline peripheral microvascular blood flow and right ventricular output or SVC flow at $24 \mathrm{~h}$. There was no influence of ductal patency on microvascular blood flow for either symptomatic ducts or physiologically significant but asymptomatic ducts. ${ }^{16}$

At $72 \mathrm{~h}$ of age, baseline microvascular blood flow continued to exhibit a significant inverse relationship with mean arterial blood pressure $\left(r^{2}=-0.4, p=0.001\right)$. However, no significant relationships were observed with microvascular blood flow or with any functional echocardiographic measure of cardiovascular function. By $120 \mathrm{~h}$ of postnatal age, the relationship between baseline microvascular blood flow and mean arterial pressure was no longer evident.

\section{DISCUSSION}

This is the first study to show that laser Doppler measurements of baseline peripheral microvascular blood flow in premature infants exhibit significant relationships with not only clinical illness severity but measures of cardiovascular function in the immediate postnatal period. Blood pressure is the product of cardiac output and systemic vascular resistance and is determined predominantly by gestational age and birth weight in the newborn period. ${ }^{17}$ The traditional understanding of the physiological basis for the development of hypotension in the preterm infant has focused on the inability of the immature myocardium to respond to increases in afterload secondary to increased systemic vascular resistance. The contribution of microvascular tone to overall vascular resistance is unclear, but our data suggest that abnormal peripheral microvascular tone, characterised by inappropriate vasodilatation, contributes to cardiovascular compromise observed after preterm birth. 


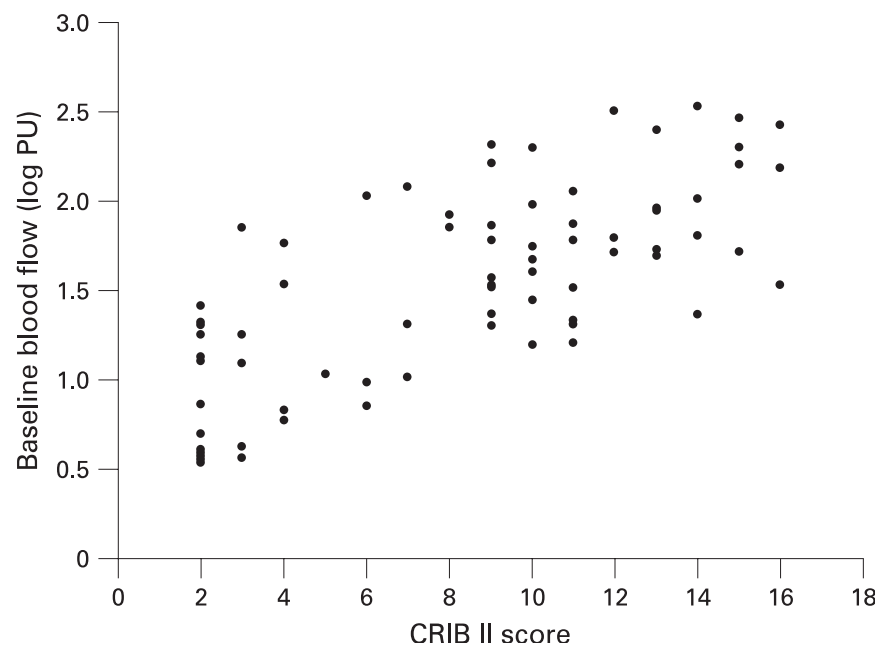

Figure 1 Relationship between baseline microvascular blood flow (log PU) at $24 \mathrm{~h}$ of age and clinical illness severity determined by the CRIB II score in infants of $\leqslant 32$ weeks' gestation $(n=74)$. $r^{2}=0.442, p<0.001$ multiple linear regression controlling for gestational age.

The preterm infant exhibits a markedly different circulatory transition after birth from that of the term infant. Recent interest has focused on novel measures of systemic blood flow ${ }^{18}$ and their relationship with the sudden increase in peripheral vascular resistance observed immediately after birth in an attempt to effectively identify those preterm infants where the transition from in utero to ex utero circulation fails. It has previously been accepted that abnormal peripheral vascular vasoregulation, characterised by inappropriately low peripheral vascular resistance, is more prevalent beyond the transitional period. ${ }^{19}$ Our data show that dysregulation of microvascular tone, with high baseline microvascular blood flow, is related to measures of cardiovascular function and reflected by the degree of clinical physiological stability in the immediate newborn period and may influence the transitional circulation. Our failure to show low flow as a risk factor, as has previously been seen with SVC, may be related to either different ventilatory practices or timing of the studies. Most of our infants were receiving continuous positive airway pressure support with a consequently lower intrathoracic pressure, a known risk factor for lower cardiac output. ${ }^{20}$ Our studies were performed at $24 \mathrm{~h}$, whereas the studies showing low SVC flow were most sensitive at 3-11 h of age, with only $3 \%$ of infants exhibiting low flow at $24 \mathrm{~h} .{ }^{14}$ This is, of course, not incompatible with our observed link with severity, as combinations of low flow followed by high flow are known to be particularly poor markers in some neonatal systems - for example, the brain. ${ }^{21}$

Data on the relationship between cutaneous haemodynamics and measures of cardiovascular function are limited and confined to the human adult and animal literature. Reductions in both cutaneous blood flow velocity and flow have been shown to precede and predict subsequent systemic haemodynamic events in animal models of haemorrhagic shock. ${ }^{22}$ Spontaneous changes in arteriovenous blood flow have been shown to be accompanied by inverse fluctuations in mean blood pressure, with elevations in heart rate and cardiac output occurring with vasoconstriction in adulthood in humans. ${ }^{23}$

Although increases in peripheral blood flow in the newborn infant, as measured by plethysmography, are associated with a rise in heart rate, ${ }^{24}$ the effect of these changes on the cardiovascular system has not previously been definitively

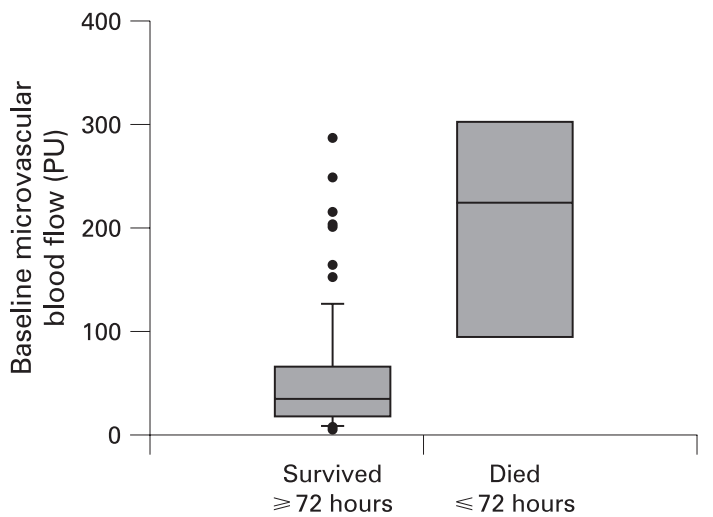

Figure 2 Baseline microvascular blood flow at $24 \mathrm{~h}$ of age in those infants who died $\leqslant 72 \mathrm{~h}$ after birth $(\mathrm{n}=8)$ and those who survived for $\geqslant 72 \mathrm{~h}(\mathrm{n}=78)$ (median (SD) 224.7 (106.2) vs 34.1 (57.6) PU; $\mathrm{p}=0.001$, Mann-Whitney U test).

studied. Within the neonatal population, capillary refill time, a crude clinical measure of peripheral microvascular perfusion, has been shown to correlate significantly with echocardiographically derived cardiac index but not mean arterial blood pressure or heart rate. ${ }^{25}$ Where temporal changes in peripheral microvascular blood flow and vasomotor activity have been determined in preterm, very-low-birth-weight infants, with respect to heart rate and blood pressure, small sample size prevents useful interpretation of the data. ${ }^{26}$ It is clear, however, that peripheral microvascular blood flow, as determined by a variety of methods, is greater in preterm than term infants ${ }^{5}$ and falls significantly in the immediate newborn period. ${ }^{39}$

Our study is limited by both the use of a single technique and assessment of the perfusion of only one body system, the skin. Laser Doppler is accepted as one of the best ways to assess the microcirculation in vivo, but, although it is particularly suited to the neonatal period because of its non-invasive nature, it does have its limitations, which are well discussed elsewhere. ${ }^{27}$ Further studies using different techniques such as peripheral near infrared spectroscopy or videomicroscopy are warranted in this population. Is the skin relevant as a representative

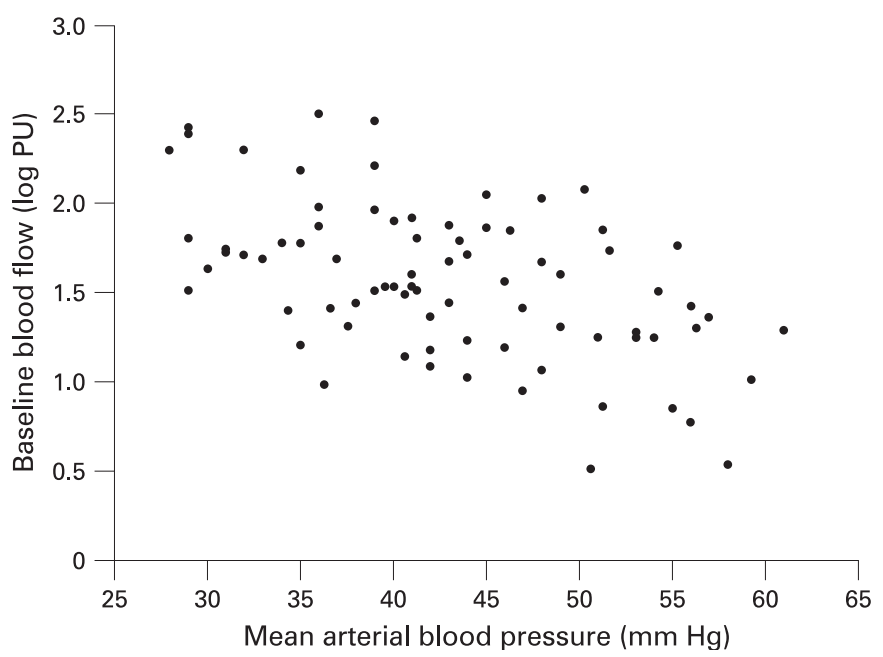

Figure 3 Relationship between baseline microvascular blood flow (log $\mathrm{PU})$ at $24 \mathrm{~h}$ of age and mean arterial blood pressure $(\mathrm{mm} \mathrm{Hg})$. $r^{2}=-0.563, p<0.001$, multiple linear regression controlling for gestational age. 


\section{What is already known on this topic}

- Peripheral microvascular blood flow is subjected to considerable change in the first days of extrauterine life.

- Peripheral microvascular blood flow is greater in preterm than term infants.

\section{What this study adds}

- Microvascular blood flow in premature infants exhibits significant relationships with clinical illness severity.

- Microvascular blood flow in premature infants exhibits significant relationships with cardiovascular function in the immediate postnatal period.

circulation? It is of note that our echocardiographically derived systemic vascular resistance correlated significantly with the measured blood flow in the skin, suggesting that the changes we were seeing were of cardiovascular relevance.

This study shows developmental changes in peripheral microvascular blood flow and the relationships between it and measures of clinical illness severity and cardiovascular function. Deviations in microcirculatory function may contribute to cardiovascular maladaptation in early neonatal life. Cardiovascular maladaptation and the resultant hypotension are common problems in the preterm infant, yet there is little clinical outcome-based evidence showing improvement in short or long term morbidity and mortality in response to commonly used treatments. ${ }^{28}$ Therefore, the importance of the microvasculature to cardiovascular adaptation after preterm birth warrants detailed study in infants at greatest risk of morbidity and mortality.

Funding: Grants from John Hunter Hospital Charitable Trust and Hunter Children's Research Foundation. MJS was supported by grants from University of Newcastle, Paediatric Research Society (UK) and the Emlyn and Jennie Thomas Cardiovascular Research Scholarship.

Competing interests: None declared.

\section{REFERENCES}

1. Brück K, Brück M, Lentis $H$. Temperature regulation in the newborn infant. Biol Neonate, 1961;3:65-119.

2. $\mathbf{O} \mathbf{W}$, Lind J. Body temperature of the newborn infant in relation to placental infusion. Acta Paediatr Scand 1967;Suppl 172:137-45.
3. Wu PYK, Wong WH, Guerra G. Peripheral blood flow in the neonate. Changes in total, skin, and muscle blood flow with gestational and postnatal age. Pediatr Res 1980;14:1374-8.

4. Martin H, Norman M. Skin microcirculation before and after local warming in infants delivered vaginally or by caesarean section. Acta Paediatr Scand 1997;86:261-7.

5. Beinder $\mathbf{E}$, Trojan A, Bucher HU, et al. Control of skin blood flow in pre- and full-term infants. Biol Neonate 1994;65:7-15.

6. Poschl JM, Weiss T, Fallahi F, et al. Reactive hyperemia of skin microcirculation in septic neonates. Acta Paediatr 1994;83:808-11.

7. Norman M, Fagrell B, Herin P. Skin microcirculation in neonatal polycythemia and effects of haemodilution. Interaction between haematocrit, vasomotor activity and perfusion. Acta Paediatr Scand 1992;82:672-7.

8. Serwer GA. Postnatal circulatory adjustments. In: Polin RA, Fox WW, Abman A, eds Fetal and neonatal physiology. Boca Raton: CRC Press, 1991.

9. Suchies HE, Brouwer C, Aarnoudse JG, et al. Skin blood flow changes, measured by laser Doppler flowmetry, in the first week after birth. Early Hum Dev 1990;23:1-8.

10. Waxman K, Police AM, Cheung CK, et al. Laser doppler velocimetry as a monitor of cardiac output changes in dogs. Crit Care Med 1985;13:194-6.

11. Swetnam SM, Yabeck SM, Alverson DC. Hemodynamic consequences of neonatal polycythemia. J Pediatr 1987;110:443-7.

12. Kubli S, Waeber B, Dalle-Ave A, et al. Reproducibility of laser doppler imaging of skin blood flow as a tool to assess endothelial function. J Cardiovasc Pharmacol 2000;36:640-8.

13. Hu J, Norman M, Wallensteen $\mathrm{M}$, et al. Increased large arterial stiffness and impaired acetylcholine induced skin vasodilatation in women with previous gestational diabetes mellitus. Br J Obstet Gynaecol 1998;105:1279-87.

14. Kluckow M, Evans N. Low systemic blood flow in the preterm infant. Semin Neonatol 2001;6:75-84.

15. Parry G, Tucker J, Tarnow-Mordi W. CRIB II: an update of the clinical risk index for babies. Lancet 2003;361:1789-91.

16. Yanowitz TD, Yao AC, Pettigrew KD, et al. Postnatal hemodynamic changes in verylow-birthweight infants. J Appl Physiol 1999;87:370-80.

17. Nuntnarumit $\mathbf{P}$, Yang W, Bada-Ellzey HS. Blood pressure measurements in the newborn. Clin Perinatol 1999;26:981-96.

18. Kluckow M, Evans N. Superior vena cava flow in newborn infants: a novel marker of systemic blood flow. Arch Dis Child Fetal Neonatal Ed 2000;82:F182-7.

19. Noori S, Seri I. Pathophysiology of newborn hypotension outside the transitional period. Early Hum Dev 2005;81:399-404.

20. Evans N, Kluckow M. Early determinants of right and left ventricular output in ventilated preterm infants. Arch Dis Child Fetal Neonatal Ed 1996;74:88-94.

21. van Bel F, Dorrepaal CA, Benders MJ, et al. Changes in cerebral hemodynamics and oxygenation in the first 24 hours after birth asphyxia. Pediatrics 1993;92:365-72.

22. Ovadia Z, Kornowski R, Gavish B, et al. Non-invasive evaluation of microcirculatory hemodynamic changes during haemorrhage followed by saline or blood transfusion. Shock 1995;4:96-101.

23. Lossius K, Eriksen M, Walloe L. Fluctuations in blood flow to acral skin in humans: connection with heart rate and blood pressure variability. J Physiol 1993;460:64155.

24. Wu PKY, Hodgman JE. Insensible water loss in premature infants: changes with postnatal development and non-ionising radiant energy. Pediatrics 1974;54:704.

25. Wodey $\mathbf{E}$, Pladys $P$, Bétrémieux $P$, et al. Capillary refilling time and hemodynamics in neonates: a Doppler echocardiographic evaluation. Crit Care Med 1998;26:1437-40.

26. Poschl J, Weiss T, Diehm C, et al. Periodic variations in skin perfusion in full term and preterm neonates using laser doppler technique. Acta Paediatr Scand 1991;:80:999-1007.

27. Obeid AN, Barnett NJ, Dougherty G, et al. A critical review of laser Doppler flowmetry. J Med Eng Technol 1990;14:178-81.

28. Evans N. Which inotrope for which baby? Arch Dis Child Fetal Neonatal Ed 2006;91:213-20.

\section{Let us assist you in teaching the next generation}

Figures from all articles on our website can be downloaded as a PowerPoint slide. This feature is ideal for teaching and saves you valuable time. Just click on the image you need and choose the "PowerPoint Slide for Teaching" option. Save the slide to your hard drive and it is ready to go. This innovative function is an important aid to any clinician, and is completely free to subscribers. (Usual copyright conditions apply.) 